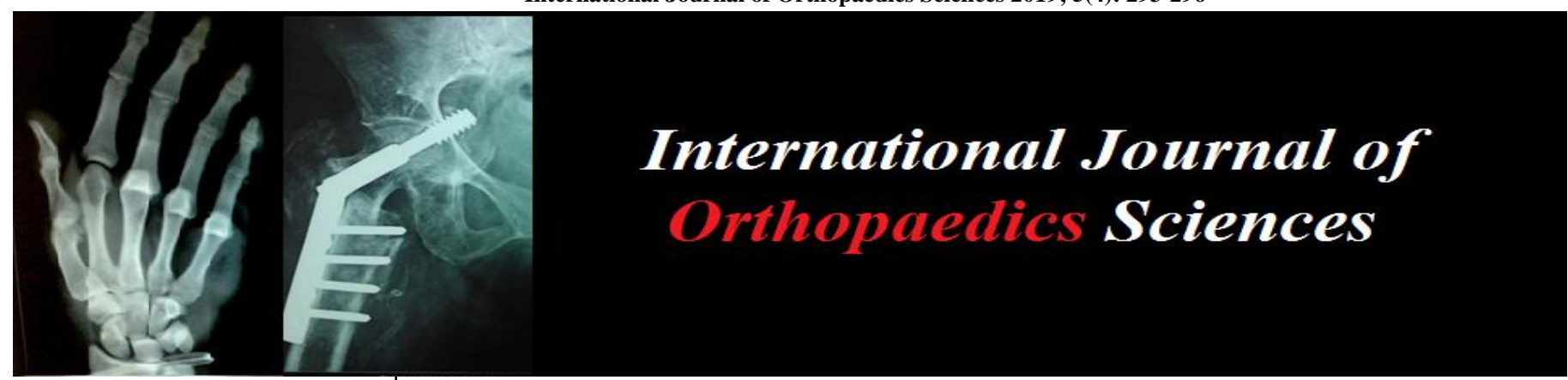

E-ISSN: 2395-1958

P-ISSN: 2706-6630

IJOS 2019; 5(4): 293-296

(C) 2019 IJOS

www.orthopaper.com

Received: 04-08-2019

Accepted: 06-09-2019

Mantoo Sayar Ahmad Bone and Joints Surgery Hospital GMC, Srinagar, Jammu and Kashmir, India

Irshad A Ganie

Bone and Joints Surgery Hospital GMC, Srinagar,

Jammu and Kashmir, India

Shafeeq Ahmad

Bone and Joints Surgery Hospital GMC, Srinagar,

Jammu and Kashmir, India

Corresponding Author: Irshad A Ganie

Bone and Joints Surgery

Hospital GMC, Srinagar,

Jammu and Kashmir, India

\section{Comparative study between the efficacy of platelet-rich plasma therapy and corticosteroid injection in the treatment of planter fasciitis}

\author{
Mantoo Sayar Ahmad, Irshad A Ganie and Shafeeq Ahmad
}

DOI: https://doi.org/10.22271/ortho.2019.v5.i4f.1687

\section{Abstract}

Background: Planter fasciitis is the common cause of heel pain and is the disabling disease in its chronic form. Various treatment modalities are available including medical therapies and surgical procedures. This study was designed to compare the efficacy of PRP therapy with corticosteroid injection in the treatment of chronic planter fasciitis.

Methods: Total of 40 patients with chronic plantar fasciitis were included in the study and were divided into 2 groups. In the PRP group $(\mathrm{n}=20), 3 \mathrm{ml}$ PRP preparation was made from the patient's blood and injected in a single dose. In the steroid group $(\mathrm{n}=20)$, a single dose of $2 \mathrm{ml}(40 \mathrm{mg})$ methylprednisolone was given. Clinical evaluation was made using the American Foot and Ankle Score (AFAS) and the visual analog scale (VAS).

Results: Both groups were similar in terms of age, gender and side involvement. Mean preinjection VAS, AOFAS scores in steroid and PRP group were $8.1 \pm 1.2,68.1 \pm 9.5$ and $7.9 \pm 1.2,67.5 \pm 9.2$ respectively. Post injection the scores improved in both the groups, with slightly more improvement of scores in PRP group than in steroid group, however no significant difference in scores of VAS and AOFAS could be detected between the two groups.

Conclusion: The local injection with steroid or PRP preparation was equally effective in terms of pain and functional outcomes in the treatment of chronic planter fasciitis.

Level of evidence: II Prospective randomized clinical trail.

Keywords: Planter fasciitis, steroid, platelet rich plasma (PRP)

\section{Introduction}

Chronic plantar fasciitis is a common orthopaedic problem that affects $10 \%$ of the population and is the commonest cause of heel pain in adults ${ }^{[1,2,3,4]}$. The etiology of plantar fasciitis is multifactorial and poorly understood but appears to be caused due to microscopic degenerative injury and local disruption of the collagen matrix rather than a failed healing response ${ }^{[5,6]}$. Reduced ankle dorsiflexion, standing for long periods of time at work, obesity, female gender and advancing age are listed as risk factors ${ }^{[1,3]}$.

Plantar fasciitis is generally a self-limiting condition. Symptoms in 80 to $90 \%$ of cases recover within 10 to 12 months with conservative treatment ${ }^{[7]}$. Heel pain is the most common reason for presentation with associated complaints of morning pain and heel discomfort with initiation of ambulation ${ }^{[8,9]}$. Clinical findings include local tenderness and associated stiffness due to soft tissue tightness ${ }^{[9]}$.

Available treatments modalities for plantar fasciitis, such as plantar fascia stretching exercises, strapping, extracorporeal shock wave therapy, nonsteroidal anti-inflammatory drugs (NSAIDs), arch supports, and heel pads ${ }^{[10,11]}$. Corticosteroid injections are often reserved for resistant plantar fasciitis after failure of conservative non-invasive interventions. They have been shown to effectively reduce the heel pain in patients with plantar fasciitis $[4,12,13]$. However, the use of corticosteroid injections for the treatment of plantar fasciitis has been shown to be associated with rupture of plantar fascia, infection, Change in skin pigmentation, post injection flare, and fat pad atrophy ${ }^{[14,15]}$.

Local injection of platelet-rich plasma is an emerging concept in treating chronic plantar fasciitis. Platelet-rich plasma injection delivers platelets and growth factors in high 
concentrations directly to the site of injury zones to induce and accelerate healing processes ${ }^{[16]}$.

\section{Methodology}

This was the prospective clinical study, conducted at Bone and joints surgery hospital, an associate hospital of Government Medical college Srinagar. Approval was granted by the local ethics committee and informed consent was obtained from all patients participating in the study. Patients, who had been diagnosed with plantar fasciitis, treated for minimum of 3 months duration and showed no benefit from conservative treatment were included in the study. Diagnosis of planter fasciitis was made by clinical examination and radiographs of ankle were examined to rule out other heel pathologies.

Total of 40 patients were included for the study and were randomly allotted into two groups, PRP group $(n=20)$ and Steroid group $(\mathrm{n}=20)$.

For preparation of platelet-rich plasma, $27 \mathrm{ml}$ of blood was withdrawn from the cubital vein and placed in a glass tube containing $3 \mathrm{ml}$ of citrate dextrose solution (ratio 9:1). Citrate dextrose solution was used to prevent clotting. The blood was centrifuged at $3200 \mathrm{rpm}$ for 12 minutes. $3 \mathrm{ml}$ PRP preparation was obtained from the upper buffy coat.

In both groups, injection was given under strict aseptic precautions. The patients were kept in supine position with eyes covered to ensure blinding. The area to be injected was prepared with $10 \%$ povidone iodine scrub. The maximum tender spot over the medial aspect of heel was marked and was anaesthetised by using 2 to $3 \mathrm{ml}$ of $2 \%$ lignocaine. $3 \mathrm{ml}$ PRP preparation was injected in the PRP group and $2 \mathrm{ml}$ (40mg) of methylprednisolone was injected in steroid group using peppering technique ${ }^{17}$ (single skin portal and 4-5 penetrations of the plantar fascia) in both groups.

After the injection, patients were advised to apply ice for pain relief if required and to continue to wear comfortable shoes with cushions and were instructed not to use NSAIDs after the procedure. All patients had physical therapy to stretch the calf muscle and plantar fascia.

Clinical assessment was made prior to the injection and at 1 month, 3 months and 6 months following the injection. Clinical evaluation included pain assessment using visual analog scale (VAS) from 0 to 10 (0 reflects absence of pain, 10 indicates the worst imaginable pain) and the functional outcome score was measured by the American Orthopaedic Foot \& Ankle Society (AOFAS) ankle-Hindfoot scale.

Results were stated as mean \pm standard deviation (SD). The comparison of normally distributed continuous variables between the groups was performed by using the Student $t$ test. Nominal categorical data between the groups were compared using chi-square test or Fisher exact test as appropriate and non-nominal distributed continuous variables were compared using the Mann-Whitney $\mathrm{U}$ test. A value of $p<0.05$ was accepted as statistically significant.

\section{Results}

Both groups were similar in terms of age, gender and side involvement as shown in table 1.The mean initial or Preinjection VAS and AOFAS scores in the steroid and PRP group was $8.1 \pm 1.2, \quad 62.7 \pm 9.5$ and $8.3 \pm 1.2, \quad 62.6 \pm 9.2$ respectively and were comparable $(p \geq 0.05)$. Post injection, the score improved considerably in each group on each follow-up; however no significant difference could be detected between the scores of the two groups at the 1, 3 and 6-month follow-ups (Table 2 and figures 1, 2).

Table 1: $\mathrm{SD}=$ standard deviation

\begin{tabular}{|c|c|c|c|}
\hline & Steroid group $($ Mean \pm SD) & PRP group $($ Mean \pm SD) & P valve \\
\hline Age (years) $($ Mean \pm SD) & $40 \pm 5.5$ & $39 \pm 5.3$ & $\geq 0.05$ \\
\hline Male/female & $6 / 14$ & $7 / 13$ & $\geq 0.05$ \\
\hline Affected foot-right/left & $11 / 9$ & $10 / 10$ & $\geq 0.05$ \\
\hline
\end{tabular}

Table 2: $\mathrm{SD}=$ standard deviation, $\mathrm{VAS}=$ visual analog scale, $\mathrm{AOFAS}=$ American Orthopaedic Foot \& Ankle Society (AOFAS) ankle-Hind foot scale.

\begin{tabular}{|c|c|c|c|}
\hline & Steroid group (Mean \pm SD) & PRP group $($ Mean \pm SD) & P valve \\
\hline Pre-injection & \multicolumn{2}{|c|}{} & \\
\hline VAS & $8.1 \pm 1.2$ & $8.3 \pm 1.2$ & $\geq 0.05$ \\
\hline AOFAS & $62.7 \pm 9.5$ & $63.6 \pm 9.2$ & $\geq 0.05$ \\
\hline \multicolumn{3}{|c|}{ Post-injection } & $\geq 0.05$ \\
\hline 1 month & $4.2 \pm 1.5$ & $3.3 \pm 0.8$ & $\geq 0.05$ \\
\hline VAS & $80.7 \pm 10.8$ & $82.9 \pm 11.2$ & $\geq 0.05$ \\
\hline AOFAS & $2.5 \pm 1.2$ & $1.9 \pm 0.8$ & $\geq 0.05$ \\
\hline 3 month & $87.7 \pm 12.2$ & $89.3 \pm 13.0$ & $\geq 0.05$ \\
\hline VAS & & & $\geq 0.05$ \\
\hline AOFAS & $2.1 \pm 0.8 .1$ & $1.5 \pm 0.5$ & $92.5 \pm 12.2$ \\
\hline 6 month & $88.9 \pm 12.7$ & & \\
\hline VAS & & & \\
\hline AOFAS & & & \\
\hline
\end{tabular}

\section{Discussion}

Chronic refractory planter fasciitis is the disabling condition.

Non- invasive treatment options like planter fascia stretching exercises, extracorporeal shock wave therapy, arch supports, heel pads and nonsteroidal anti-inflammatory drugs (NSAIDs) are usually less effective in chronic refractory cases $[4,10,11]$. The use of steroid injections for plantar fasciitis has been reported in literature and is found to be useful in both short

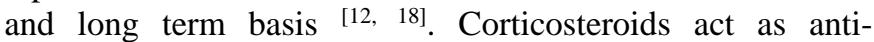
inflammatory agents and have been shown to inhibit 
fibroblast proliferation and expression of ground substance proteins ${ }^{[13]}$. However, steroid injections have been reported to be related to plantar fascia tear, fat pad atrophy, abscess, and osteomyelitis ${ }^{[14,15]}$.

Recently, it has been reported that planter fasciitis is a degenerative disorder rather than an inflammatory process $[5,6$, ${ }^{19]}$. Degeneration of collagen occurs at the site of the lesion because of micro tears of the fascia that do not heal. This observation was further supported by histological examination of the plantar fascia obtained during surgery of chronic planter fasciitis patients, which shows no inflammatory cell invasion at the site of the lesion, the normal fascia and surrounding tissue was replaced by Angiofibroblastic hyperplasic tissue ${ }^{[5]}$.

PRP, being a concentrate of platelets that are a source of autologous growth factors such as insulin like growth factor-1 (IGF-1), transforming growth factor $\beta$ (TGF- $\beta$ ), vascular endothelial growth factor (VEGF), platelet derived growth factor (PDGF) and fibroblast growth factor (FGF), helps in cellular migration, synthesis of collagen, and angiogenesis and thus helps in tendon and ligament healing [16, 20, 21, 22]. Several studies have reported the use of PRP as safe and effective treatment option in chronic refractory plantar fasciitis.

Monto ${ }^{[23]}$ found that platelet-rich plasma injection was more effective and durable than corticosteroid injection at 2 years of follow-up in a study of 40 patients. Shetty et al. [24] compared the effectiveness of platelet-rich plasma and corticosteroid injections in 60 patients and found no significant difference at 6 months of follow-up. Aksahin et al. [25] compared intralesional corticosteroid and platelet-rich plasma injections for plantar fasciitis, the treatments were found to be equally effective. Lee and Ahmad ${ }^{[26]}$ compared intralesional autologous blood injection with corticosteroid injection in patients with chronic plantar fasciitis. At 6 weeks and 3 months of follow-up, the corticosteroid group had significantly lower visual analog scale scores than the autologous blood group, but the difference was not significant at 6 months.

The current study found that local platelet-rich plasma and corticosteroid injections were effective at 1, 3 and 6 months of follow-up, with significant improvement in visual analog scale and AOFAS scores. Comparison of platelet-rich plasma and corticosteroid injections showed no significant difference at each follow up, however, platelet-rich plasma injection had better VAS and AOFAS scores compared with corticosteroid injection. No local or systemic complications were seen in any patient during the application or follow-up.

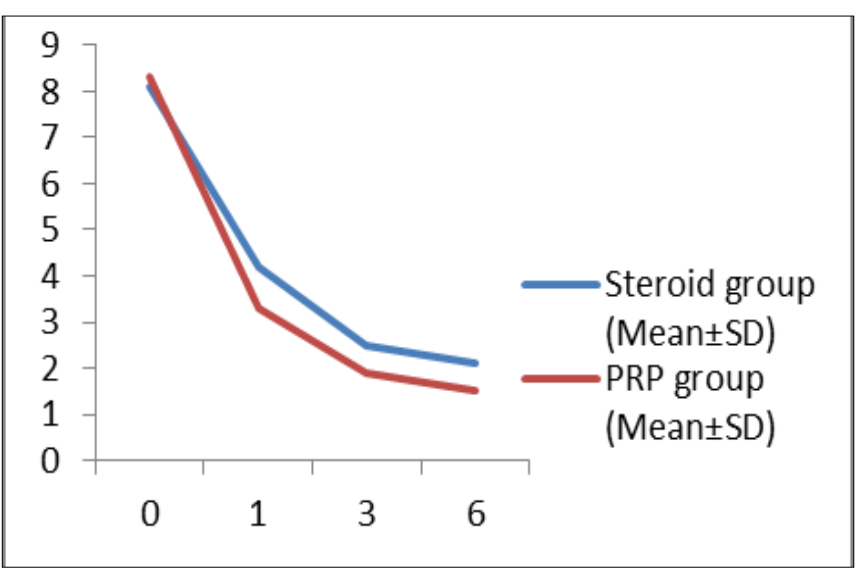

Fig 1: VAS scores

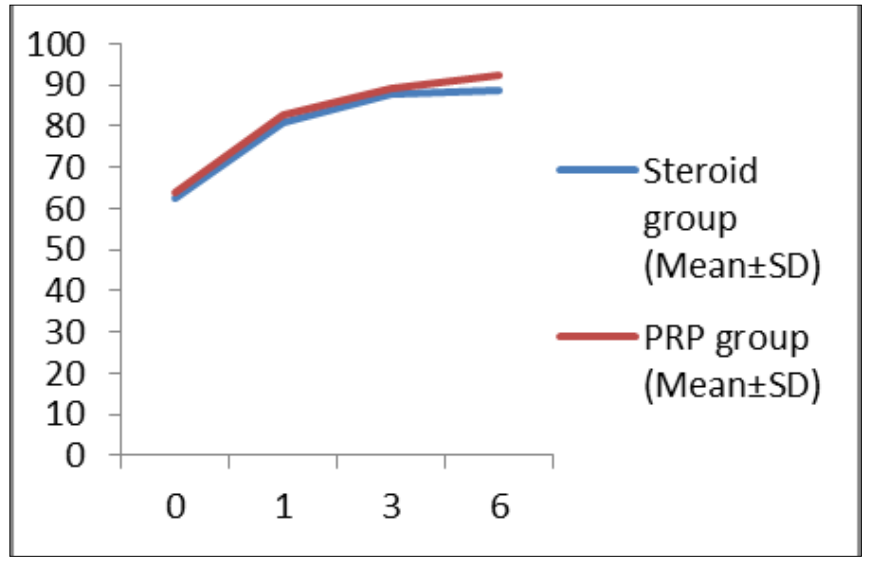

Fig 2: AOFAS scores

\section{Conclusion}

The observations made in the present study suggest that the treatment of plantar fasciitis with steroid or PRP injection is equally effective.

\section{References}

1. Riddle DL, Pulsic M, Pidcoe P, Johnson RE. Risk factors for plantar fasciitis: A matched case-control study. J Bone Joint Surg Am. 2003; 85:872-877.

2. Riddle DL, Shappert SM. Volume of ambulatory care visits and patterns of care for patients diagnosed with plantar fasciitis: A national study of medical doctors. Foot Ankle Int. 2004; 25:303-310.

3. Scher DL, Belmont PJ Jr, Bear R et al. The incidence of plantar fasciitis in the United States military. J Bone Joint Surg Am. 2009; 91:2867-2872.

4. Crawford F, Thomson C. Interventions for treating plantar heel pain. Cochrane Database Syst Rev. 2010; 1:CD000416

5. Jarde O, Diebold P, Havet E, Boulu G, Vernois J. Degenerative lesions of the plantar fascia: surgical treatment by fasciectomy and excision of the heel spur. A report on 38 cases. Acta Orthop Belg. 2003; 69:267-74.

6. Lemont H, Ammirati KM, Usen N. Plantar fasciitis: A degenerative process (Fasciosis) without inflammation. J Am Podiatr Med Assoc. 2003; 93:234-7.

7. Davis PF, Severud E, Baxter DE. Painful heel syndrome: results of Nonoperative treatment. Foot Ankle Int. 1994; 15:531-5.

8. League AC. Current concepts review: plantar fasciitis. Foot Ankle Int. 2008; 29:358-66.

9. Toomey EP. Plantar heel pain. Foot Ankle Clin. 2009; 14:229-245

10. Healey K, Chen K. Plantar fasciitis: current diagnostic modalities and treatments. Clin Podiatr Med Surg. 2010; 27:369-80.

11. Gill LH, Kiebzak GM. Outcome of nonsurgical treatment for plantar fasciitis. Foot Ankle Int. 1996; 17:527-32.

12. Genc H, Saracoglu M, Nacir B, Erdem HR, Kacar M. Long term ultrasonographic follow-up of plantar fasciitis patients treated with steroid injection. Jt Bone Spine. 2005; 72(1):61-65

13. McMillan AM, Landorf KB, Gilheany MF, Bird AR, Morrow AD, Menz HB. Ultrasound guided injection of dexamethasone versus placebo for treatment of plantar fasciitis: protocol for a randomised controlled trial. J Foot Ankle Res. 2010; 3:15.

14. Acevedo JI, Beskin JL. Complications of plantar fascia rupture associated with corticosteroid injection. Foot 
Ankle Int. 1998; 19(2):91-97

15. Sellman JR. Plantar fascia rupture associated with corticosteroid injection. Foot Ankle Int. 1994; 15(7):376381.

16. Molloy T, Wang Y, Murrell G. The roles of growth factors in tendon and ligament healing. Sports Med. 2003; 33(5):381-394.

17. Kalaci A, Cakici H, Hapa O, Yanat AN, Dogramaci Y, Sevinc TT. Treatment of plantar fasciitis using four different local injection modalities: a randomized prospective clinical trial. J Am Podiatr Med Assoc. 2009; 99(2):108-113.

18. Crawford F, Atkins D, Young P, Edwards J. Steroid injection for heel pain: evidence of short-term effectiveness. A randomized controlled trial. Rheumatology (Oxford). 1999; 38(10):974-977.

19. Buchbinder R. Clinical practice: plantar fasciitis. N Engl J Med. 2004; 350 (21):2159-2166

20. Anitua E, Andia I, Sanchez M et al. Autologous preparations rich in growth factors promote proliferation and induce VEGF and HGF production by human tendon cells in culture. J Orthop Res. 2005; 23:281-286

21. Bendinelli P, Matteucci E, Dogliotti G et al. Molecular basis of anti-inflammatory action of platelet-rich plasma on human chondrocytes: mechanisms of NF-(ordM) B inhibition via HGF. J Cell Physiol. 2010; 225:757-766.

22. Eppley BL, Woodell JE, Higgins J. Platelet quantification and growth factor analysis from platelet-rich plasma: implications for wound healing. Plast Reconr Surg. 2004; 114:1502-1508

23. Monto RR. Platelet-rich plasma efficacy versus corticosteroid injection treatment for chronic severe plantar fasciitis. Foot Ankle Int. 2014; 35:313-318.

24. Shetty VD, Dhillon M, Hegde C, Jagtap P, Shetty S. A study to compare the efficacy of corticosteroid therapy with platelet-rich plasma therapy in recalcitrant plantar fasciitis: a preliminary report. Foot Ankle Surg. 2014; 20(1):10-13.

25. Aksahin E, Dogruyol D, Yüksel HY, et al. The comparison of the effect of corticosteroids and plateletrich plasma (PRP) for the treatment of plantar fasciitis. Arch Orthop Trauma Surg. 2012; 132:781-785.

26. Lee TG, Ahmad TS. Intralesional autologous blood injection compared to corticosteroid injection for treatment of chronic plantar fasciitis: a prospective, randomized, controlled trial. Foot Ankle Int. 2007; 28:984-990. 